\title{
Molecular differentiation of common promoters in Salmonella class 1 integrons
}

\author{
Tae-Eun Kim ${ }^{\text {a }}$, Hyuk-Joon Kwon ${ }^{\text {b, }}$, Sun-Hee Cho ${ }^{\text {a,e }}$, Shukho Kim ${ }^{\mathrm{f}}$, Bok-Kwon Lee ${ }^{\mathrm{f}}$, \\ Han-Sang Yoo ${ }^{\mathrm{b}, \mathrm{c}, \mathrm{e}}$, Yong-Ho Park ${ }^{\mathrm{b}, \mathrm{d}, \mathrm{e}}$, Sun-Joong Kim ${ }^{\mathrm{a}, \mathrm{b}, \mathrm{e}}$ \\ a Laboratory of Avian Diseases, Seoul National University, Seoul, 151-742, South Korea \\ ${ }^{\mathrm{b}}$ Zoonotic Disease Institute (ZooDI), Seoul National University, Seoul, 151-742, South Korea \\ ${ }^{\mathrm{c}}$ Laboratory of Infectious Diseases, Seoul National University, Seoul, 151-742, South Korea \\ d Department of Microbiology, Seoul National University, Seoul, 151-742, South Korea \\ e College of Veterinary Medicine and BK21 for Veterinary Science, Seoul National University, Seoul, 151-742, South Korea \\ ${ }^{\mathrm{f}}$ Department of Microbiology, Korea Center for Disease Control and Prevention, Seoul, 122-701, South Korea
}

Received 17 May 2006; received in revised form 14 September 2006; accepted 28 September 2006

Available online 22 November 2006

\begin{abstract}
The integron is a mobile gene element which harbors antibiotic-resistance gene cassettes capable of site-specific integration. Among the four known types of integrons, the class 1 integron has been associated with multidrug-resistance in pathogenic bacteria. These gene cassettes have been the focus of a series of studies. The gene cassettes share a common promoter, and their expression levels are affected not only by their proximity to the promoter, but also by the strength (weak, hybrid and strong) of the common promoter, P1, as well as the presence of the additional promoter, P2. In this study, we developed molecular methods for the differentiation of promoter structures using PCR, restriction enzyme analysis, and polyacrylamide gel electrophoresis, and have applied them to the characterization of class 1 integrons in 33 non-typhoidal Salmonella serotypes in Korea. Class 1 integrons were detected in four serotypes: $S$. Derby (SD), $S$. Istanbul (SI), $S$. Paratyphi B (SPB), and $S$. Livingstone (SL), and the amplicon sizes were $1.0 \mathrm{~Kb}(\mathrm{SD}, \mathrm{SI}$ and $\mathrm{SPB})$ and $2.0 \mathrm{~Kb}(\mathrm{SL})$. All of the $1.0 \mathrm{~kb}$ amplicons harbored gene cassettes (aadA1 or

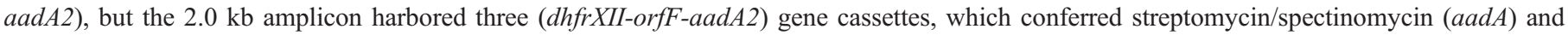
trimethoprim $(d h f r)$ resistances. Our promoter structure study revealed three types of promoters; strong P1 (SD), weak P1 (SPB and SL), and weak $\mathrm{P} 1+\mathrm{P} 2$ (SI). In conclusion, the class 1 integrons were detected in Korean NTS, and their promoter structures were found to be variable. Therefore, our methods may prove helpful in terms of our understanding of molecular diversity, as well as the transmission of class 1 integrons and phenotype-genotype relationships in antibiotic-resistance.
\end{abstract}

(C) 2006 Elsevier B.V. All rights reserved.

Keywords: Class 1 integron; Non-typhoidal Salmonella; Antibiotic-resistance; Promoter

\section{Introduction}

In the mid-1980s, the DNA sequencing of several seemingly unrelated antibiotic-resistance-associated genes revealed common upstream and downstream regions (Cameron et al., 1986; Hall and Vocker, 1987). The upstream harbored the attI site, a

\footnotetext{
* Corresponding author. Seoul National University, Laboratory of Influenzavirus, Zoonotic Disease Institute (ZooDI), San 56-1, Shillim-Dong, GwanakGu, Seoul, 151-742, South Korea. Tel.: +82 2880 1288; fax: +82 28856614 .

E-mail address: kwonhj01@snu.ac.kr (H.-J. Kwon).
}

common promoter sequence, $\mathrm{P}_{\text {ant }}(\mathrm{P} 1)$, and the opposite strand, the integrase gene (int) (Hall and Collis, 1995; Recchia and Hall, 1995), whereas the 3 '-conserved segment harbored an antiseptic resistance gene (qacE1), a sulphonamide resistance gene (sull), and an open reading frame (orf5) of undetermined function (Hall and Stokes, 1993). The central variable region included varying combinations of inserted gene cassettes. Thus far, more than 60 different cassettes associated with resistance genes have been characterized, which consequently renders their bacterial hosts resistant to a broad spectrum of antimicrobial agents (Recchia and Hall, 1995). This genetic element lacks direct or indirect 
Table 1

Salmonella serotypes used in this study and their antibiotic-resistances

\begin{tabular}{|c|c|c|c|}
\hline Serotype(serogroup) & Antibiotic resistance $^{a}$ & Serotype & Antibiotic resistance \\
\hline S. Agama (B) & $\mathrm{S}$ & S. Montevideo(C1) & \\
\hline S. Bardo(C3) & & S. Muenchen (C2) & AM, S, Te, SAM (I) K, TIC, NA \\
\hline S. Bareilly(C1) & & S. Nchanga (E1) & $\mathrm{AM}, \mathrm{S}, \mathrm{CF}, \mathrm{FOX}, \mathrm{CRO}$ \\
\hline$S$. Blockley (C2) & Te, K, TIC, NA & S. Newport(C2) & \\
\hline S. Bradford (B) & & S. Nigeria(C1) & $\mathrm{S}$ \\
\hline S. Braenderup (C1) & & S. Othmarchen (C1) & $\mathrm{Te}$ \\
\hline$S$. Brezany (B) & $\mathrm{S}$ & S. Paratyphi A (A) & NA \\
\hline$S$. Derby(B) & $\mathrm{S}, \mathrm{Te}$ & $S$. Paratyphi B(B) & $\mathrm{AM}, \mathrm{C}, \mathrm{S}, \mathrm{Te}, \mathrm{TIC}, \mathrm{NA}$ \\
\hline$S$. Give (E1) & $\mathrm{S}$ & S. Planckendael(C1) & $\mathrm{S}$ \\
\hline S. Hadar(C2) & $\mathrm{S}, \mathrm{Te}$ & $S$. Rissen $(\mathrm{C} 1)$ & $\mathrm{Te}$ \\
\hline$S$. Infantis(C1) & & S. Scenftenberg(E4) & \\
\hline S. Istanbul(C3) & $\mathrm{S}, \mathrm{Te}, \mathrm{GM}$ & S. Thompson(C1) & $\mathrm{S}$ \\
\hline$S$. Kedougou(G) & $\mathrm{S}$ & S. Tibati(E1) & \\
\hline S. Kentucky(C3) & $\mathrm{S}$ & $S$. Travis(B) & $\mathrm{Te}$ \\
\hline$S$. Lindenberg(C2) & NA & S. Tumodi(B) & $\mathrm{S}$ \\
\hline S. Livingstone(C1) & AM, C, S, SXT, Te, CP, K, TIC, GM & S. Uppsala(B) & $\mathrm{S}$ \\
\hline S. Mbandaka(C1) & & & \\
\hline
\end{tabular}

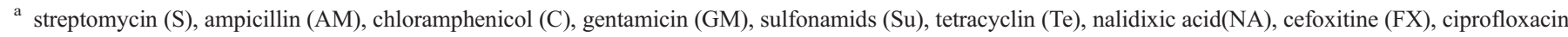

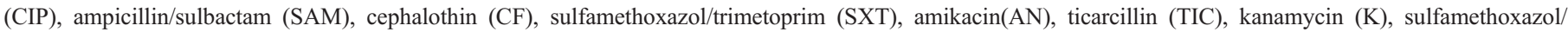
trimetoprim (SXT), cefoxitim (FOX), ceftriaxone (CRO).

repeat sequences at its ends and many gene products are associated with transposition. For this reasons, the new element was termed integron in contrast to transposon. Thus far, four types of integrons, with different int genes, have been identified, and the majority of the integrons obtained from clinical isolates are members of class 1 (Recchia and Hall, 1995; Martinez-Freijo et al., 1999; Naas et al., 2001). Class 1 integrase catalyzes sitespecific recombination between the attI of the integron and a 59base element of the gene cassette, which is mobile and harbors a variety of antibiotic-resistance genes, as well as unknown open reading frames (Recchia and Hall, 1995). Most of the gene cassettes lack their own promoters, and integrons function as natural expression vectors by supplying a common promoter, $\mathrm{P}_{\text {ant }}(\mathrm{P} 1)$, which is located in the conserved sequences upstream of the inserted genes (Hall and Stokes, 1993; Hall and Collis, 1995). Mutations in the -35 and -10 regions of $P_{\text {ant }}$ modulate levels of gene expression and the varying promoter types were classified into weak, hybrid, and strong promoters (Lévesque et al., 1994). Such single nucleotide polymorphisms (SNPs) provide selective advantages to bacteria during the course of a single infection, epidemic spread, or the long-term evolution of virulence especially if antibiotic treatment is involved (Sokurenko et al., 1999). In a few integrons a second promoter, P2, has been formed via the insertion of three $\mathrm{G}$ residues, which extends the length of DNA between the -35 and -10 boxes to 17 base pairs (Tenover et al., 1988; Schmidt et al., 1988). The expression level of the gene cassette is influenced not only by its proximity to P1 and the copy number of gene cassettes, but also by the potency of P1 and the presence or absence of P2 (Collis and Hall, 1995; Lévesque et al., 1994). Thus far, studies concerning the class 1 integrons have focused solely on the characterization of gene cassettes, and have generally ignored the structures of the promoters. In this study, we developed simple promoter characterization methods via restriction enzyme analysis (REA) and polyacrylamide gel electrophoresis (PAGE), and applied them to the characterization of the class 1 integrons in non-typhoidal Salmonella cases in Korea.

\section{Materials and methods}

\subsection{Bacteria}

33 Salmonella enterica serotypes were isolated from the stools of patients hospitalized for acute gastroenteritis in 2000, and were characterized by the Laboratory of Enteric Infections, Department of Microbiology, Korea Center for Disease Control and Prevention, located in Seoul, Korea. Salmonella was serotyped via slide agglutination tests using $\mathrm{O}$ and $\mathrm{H}$ group antisera (Difco Laboratories, Detroit, MI, USA). The serotypes employed in this study are summarized in Table 1.

\subsection{Antimicrobial susceptibility testing}

Salmonella serotypes were evaluated for their susceptibility to 18 antimicrobial agents (Table 1) via disk diffusion assay following CLSI (formerly NCCLS) guidelines (NCCLS, 2003).

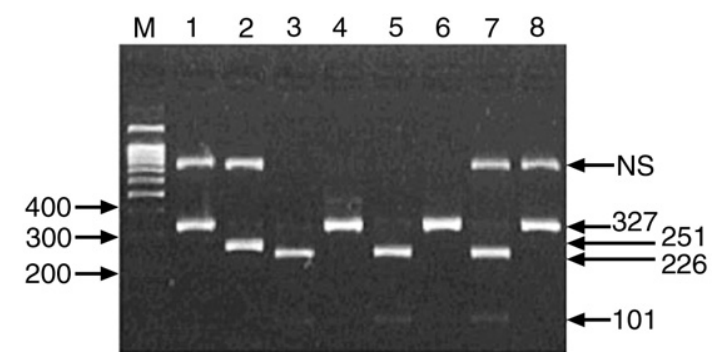

Fig. 1. Molecular typing of $\mathrm{P}_{\text {ant }}(\mathrm{P} 1)$ via restriction enzyme analysis. Lanes: $\mathrm{M}$, 100 bp molecular weight marker (iNtRON Biotechnology, Seoul, Korea); $1, S$. Derby (AluI); 2, S. Derby (HincII); 3, S. Istanbul (AluI); 4, S. Istanbul (HincII); 5, S. Paratyphi B (AluI); 6, S. Paratyphi B (HincII); 7, S. Livingstone (AluI); 8, $S$. Livingstone (HincII). 
Table 2

Genetic structures of the variable regions of class 1 integrons and antibiotics resistances

\begin{tabular}{llll}
\hline Serotypes & $\begin{array}{l}\text { Amplicon } \\
\text { size }(\mathrm{Kb})\end{array}$ & Gene cassette & Antibiotic resistance \\
\hline S. Derby & 1.0 & $\operatorname{aadA2}$ & $\mathrm{S}, \mathrm{Te}$ \\
S. Istanbul & 1.0 & $\operatorname{aadA1}$ & $\mathrm{S}, \mathrm{Te}, \mathrm{GM}$ \\
S. Paratyphi B & 1.0 & $\operatorname{aadA2}$ & $\mathrm{AM}, \mathrm{C}, \mathrm{S}, \mathrm{Te}, \mathrm{TIC}$ \\
S. Livingstone & 2.0 & $\operatorname{dhfr}$ XII-orfF-aadA2 & $\mathrm{AM}, \mathrm{C}, \mathrm{S}, \mathrm{SXT}, \mathrm{Te}$ \\
& & & $\mathrm{GM}, \mathrm{K}, \mathrm{TIC}$ \\
\hline
\end{tabular}

\subsection{Detection of class 1 integrons by PCR}

All serotypes were evaluated for the presence of class 1 integrons using primers, 5'CS and 3'CS (Lévesque et al., 1995). The total Salmonella DNA was extracted using G-spin for Bacteria kit (iNtRON Biotechnology Co., Seoul, Korea) in accordance with the manufacturer's protocol. The PCR was conducted as before (Kwon et al., 2002).

\subsection{Characterization of $P_{\text {ant }}$ by REA and polyacrylamide gel electrophoresis (PAGE)}

Proximity to the common promoter $\mathrm{P}_{\text {ant }}$ affects the expression of gene cassettes of the class 1 integrons, as does the strength of the promoter. According to the nucleotide sequence of the -35 and -10 regions of $\mathrm{P}_{\text {ant }}(\mathrm{P} 1)$ it can be divided into strong, hybrid and weak (Fig. 1). The -35 region of strong P1 and the -10 region of weak P1 are digested by HincII and AluI, respectively, but the -35 and -10 regions of hybrid $\mathrm{P} 1$ are not digested by the enzymes. In addition, three nucleotide (GGG) insertions have been shown to generate a new promoter, $\mathrm{P} 2$ downstream of $\mathrm{P}_{\text {ant }}$. For the rapid and simple characterization of the promoter structure of class 1 integrons, we have also designed PCR primer sets for REA and sequencing (IntProF: 5'-ATG CCT CGA CTT CGC TGC T-3', IntProR: 5'-ACT TTG TTT TAG GGC GAC TGC-3', amplicon size 327 bp) and for PAGE (Int PT2F: 5' TGG TAA CGG CGC AGT GGC-3', 5'-TTG CTG CTT GGA TGC CCG A-3', amplicon size 82 bp or 85 bp in the case of the three-nucleotide-insertion). The amplicons generated by the primer set (IntProF/ IntProR) were then treated with HincII and AluI in separate tubes as before (Kwon et al., 2000), and electrophoresed in 2\% agarose. In order to identify P2 (gel shift by $3 \mathrm{bp}$ insertion), we conducted PAGE with 12\% (19:1) polyacrylamide gel at $90 \mathrm{~V}$ for $4 \mathrm{~h}$, and the gel was stained with ethidium bromide.

\subsection{Sequencing of PCR products}

PCR products, representing the different amplicons generated for the 33 Salmonella serotypes, were purified via simple ethanol precipitation and sequenced as described before (Kwon et al., 2000). The obtained DNA sequences were then compared to entries in the GenBank database of the National Center for Biotechnology Information BLAST network (Altschul et al., 1990).

\subsection{Nucleotide sequence accession numbers}

Nucleotide sequences were submitted to the GenBank database and the following accession numbers were assigned: aadA1 in $S$. Istanbul (AY125351), aadA2 in $S$. Derby (AY127872), in $S$. Paratyphi B (AY125352) and in S. Livingstone (AY128094), and dhfr XII in S. Livingstone (AY127874).

\section{Results and discussion}

\subsection{Antimicrobial susceptibility}

Ten serotypes-Bardo, Bareilly, Bradford, Braenderup, Infantis, Mbandaka, Montevideo, Newport, Senftenberg and Tibati-were found to be susceptible to all tested antibiotics (Table 1). Streptomycin- and tetracycline-resistance were most frequently detected, at 50\% (16/33) and 32\% (10/33), respectively. Multi-drug-resistant (MDR) Salmonella serotypes evidencing resistance to three or more different antibiotics were reported in serotypes Typhimurium, Ohio, Panama, Brandenburg, Virchow, and Hadar (Guerra et al., 2000). In Korea, MDR $S$. Typhi, $S$. Typhimurium, and $S$. Enteritidis were reported (Yang et al., 2001; Pai et al., 2003; Kim et al., 2004), but MDR had not been previously detected in serotypes Muenchen, Paratyphi B, and Livingstone. Therefore, the appearance of MDR in these minor NTS in Korea (Table 1) makes necessary further studies into the mechanisms underlying the evolution and spread of resistance, as well as effective control methods.

Table 3

Single nucleotide polymorphisms (SNPs) of aadA2 in Korean pathogenic bacteria

\begin{tabular}{|c|c|c|c|}
\hline Bacteria & Accession no. & SNP (amino acid changes and codon number) & Gene cassettes \\
\hline \multirow[t]{2}{*}{ E. coli } & AY878717 & $-{ }^{\mathrm{a}}$ & dhfrXVI-aadA2 \\
\hline & AY878718 & GAG > GAA $\left(151^{\mathrm{b}}\right)$ & aacA4-bla oxa-4-aadA2 $_{2}$ \\
\hline \multirow[t]{2}{*}{$K$. pneumoniae } & AF180731 & GTA $>$ GAA (V3E) ATT $>$ GTT (I9V) GAC $>$ GAT $\left(69^{\text {b }}\right)$ TGG $>$ TGT $($ W211C) & dhfrXII-orfF-aadA2 \\
\hline & AF227505 & - & aacA4-oxa-1-aadA2 \\
\hline P. aeruginosa & AF418284 & CTG > CAA (L29Q) GAG > GGG (E123G) & blaVIM-2-catB8-aacA4-aadA2 \\
\hline$S$. Derby & AY127872 & GCG > ACG (A150T) & $\operatorname{aad} A 2$ \\
\hline$S$. Paratyphi B & AY125352 & - & $\operatorname{aad} A 2$ \\
\hline S. Livingstone & AY128094 & GAG＞GGG (E123G) & dhfrXII-orfF-aadA2 \\
\hline
\end{tabular}

\footnotetext{
a No SNP.
}

b Silent mutation. 
Table 4

Molecular characterization of class 1 integron common promoter

\begin{tabular}{|c|c|c|c|c|c|c|c|}
\hline \multirow[t]{2}{*}{ Promoter type } & \multicolumn{2}{|l|}{-35 Region } & \multicolumn{2}{|l|}{ Spacing } & \multicolumn{2}{|c|}{ - 10 Region } & \multirow[t]{2}{*}{ Serotypes } \\
\hline & Seq & Hinc II (GTY/RAC) & No. of nt & 3-nt-insertion & Seq & Alu I (AG/CT) & \\
\hline strong & gTTGACA & + & 17 & & TAAACT & - & $S$. Derby \\
\hline $\mathrm{P} 1$ & cTTGTTA & - & 14 & - & TACAGT & - & \\
\hline \multirow[t]{2}{*}{ weak P1 + P2 } & gTGGACA & - & 17 & & TAAGCT & + & S. Istanbul \\
\hline & cTTGTTA & - & 17 & + & TACAGT & - & \\
\hline \multirow[t]{2}{*}{ weak P1 } & gTGGACA & - & 17 & & TAAGCT & + & S. Paratyphi B \\
\hline & cTTGTTA & - & 14 & - & TACAGT & - & $S$. Livingstone \\
\hline
\end{tabular}

\subsection{Molecular characterization of gene cassettes}

Class 1 integrons were detected in four (serotypes Derby, Istanbul, Paratyphi B and Livingstone) of 33 NTS serotypes. The amplicons generated by the $5^{\prime} \mathrm{CS} / 3^{\prime} \mathrm{CS}$ primer set were approximately 1.0 and $2.0 \mathrm{~Kb}$ in size (Table 2). Nucleotide sequencing revealed aadA2 ( $S$. Derby and $S$. Paratyphi B) and aadAl ( $S$. Istanbul) in the $1.0 \mathrm{~kb}$ amplicons, and $\operatorname{dhfrXII-orfF-}$ $\operatorname{aadA2}$ (S. Livingstone) in the $2.0 \mathrm{~Kb}$ amplicon (Table 2). Such gene cassettes had already been reported in Korea (Kwon et al., 2002; Kang et al., 2005). Not all of the streptomycin-resistant NTS serotypes harbored class 1 integrons, but the streptomycin resistance of the integron-positive serotypes could be attributed to $\operatorname{aadA}$ (Tables 1 and 2). The coding regions of the aadA2 gene sequence identified in S. Derby (AY127872), S. Paratyphi B (AY125352) and $S$. Livingstone (AY128094) were compared with other $a a d A 2$ genes detected in pathogenic bacteria in Korea (AF180731, AF257505, AF418284, AY878717, and AY878718). We detected six amino-acid-changing SNPs at codons 3, 9, 29, 123, 150, and 211, and two silent SNPs at codons 69 and 151 (Table 3). Each isolate harbored zero to four SNPs, and only the SNP at codon 123 was found to exist in common in Pseudomonas aeruginosa and S. Livingstone. The AadA2 genes in Escherichia coli (AY878717, Jeong et al., 2005), Klebsiella pneumoniae (AF227505) and S. Paratyphi B harbored no SNPs, but the contents of their gene cassettes were completely different. In addition, K. pneumoniae (AF180730) and $S$. Livingstone evidenced identical contents, but their AadA2 genes differed at codons 3, 9, 69, 123, and 211 (Table 3). SNPs in the fimH of E. coli and S. Typhimurium were associated with pathogenic adaptation (Sokurenko et al., 1998; Boddicker et al., 2002). Some of the SNPs in $s p v R$ were conserved in certain serotypes of Salmonella, and they could be used in the rough differentiation of Salmonella serotypes (Kwon et al., 2004). Therefore, SNPs identified in aadA2 may prove useful as molecular markers for the study of the transmission and evolution of aadA2-bearing class 1 integrons.

Gene cassettes can be recombined into an integron, but conversely can be looped-out from an integron (Hall et al., 1991). The array of $d h f r X I I-o r f F-a a d A 2$ gene cassettes can be formed by the additional recombination of $\operatorname{dhfrXII}$ into the integron bearing the aadA2 cassette. The gene cassette most proximal to the common promoter ( $\mathrm{P} 1$ and/or $\mathrm{P} 2)$ is expressed more abundantly than others, and the localization of dhfrXII proximally to $\mathrm{P} 1$ in $S$. Livingstone represents selection pressure by trimethoprim (Hall and Collis, 1995). Resistances to tetracycline, chloramphenicol, kanamycin, gentamicin, ampicillin, and nalidixic acid can be transferred by integrons; however, in the case of the NTS serotypes studied in this work, resistance was not associated with the Class 1 integron (Recchia and Hall, 1995; Martínez-Martínez et al., 1998).

\subsection{Molecular characterization of common promoters of gene cassettes}

According to the results of a promoter study involving CAT assays, the relative strengths of the strong P1 and the weak P1+ P2 to weak P1 were approximately 32-fold and 16-fold higher, respectively (Lévesque, 1994). The amplicons generated by the primer set (IntProF/ IntProR) were digested by HincII ( $S$. Derby) or AluI (S. Istanbul, $S$. Livingstone and $S$. Paratyphi B) (Fig. 1), and gel shift via 3-nt-insertion was detected in $S$. Istanbul. Therefore, the class 1 integrons of $S$. Derby, $S$. Livingstone and $S$. Paratyphi B harbored strong and weak P1, respectively. $S$. Istanbul was found to harbor P2, as well as weak P1 (weak P1 + P2) (Table 4). All of the amplicons employed in this promoter study were sequenced, and all of the nucleotide variations were confirmed. $S$. Derby and $S$. Paratyphi B were similar with regard to the contents of the gene cassette, but were distinct in terms of the structures of the promoters. Therefore, our promoter structure assays may provide us with further insight into class 1 integron transmission and evolution, as well as the relationships between antibiotic-resistances and certain genotypes.

\section{Acknowledgements}

This work was supported by the Korea Research Foundation Grants KRF-005-E00076 and KRF-005-E00077.

\section{References}

Altschul, A.F., Gish, W., Miller, W., Myers, E.W., Lipman, D.J., 1990. Basic local alignment search tool. J. Mol. Biol. 215, 403-410.

Boddicker, J.D., Ledeboer, N.A., Jagnow, J., Jones, B.D., Clegg, S., 2002. Differential binding to and biofilm formation on, Hep-2 cells by Salmonella enterica serovar Typhimurium is dependent upon allelic variation in the fimH gene of the fim gene cluster. Mol. Microbiol. 45, 1255-1265.

Cameron, F.H., GrootObbink, D.J., Ackerman, V.P., Hall, R.M., 1986 Nucleotide sequence of the $\operatorname{ADD}(23)$ aminoglycosides adenylyltransferase determinant $a a d B$. Evolutionary relationship of this region with those surrounding aadA in R538-1 and dhfrII in R388. Nucleic Acids Res. 14, 8625-8635. 
Collis, C.M., Hall, R.M., 1995. Expression of antibiotic-resistance genes in the integrated cassettes of integrons. Antimicrob. Agents Chemother. 39, 155-162.

Guerra, B., Soto, S., Cal, S., Mendoza, M.C., 2000. Antimicrobial resistance and spread of class 1 integrons among Salmonella serotypes. Antimicrob. Agents Chemother. 44, 2166-2169.

Hall, R.M., Collis, C.M., 1995. Mobile gene cassettes and integrons: capture and spread of genes by site-specific recombination. Mol. Microbiol. 15, 593-600.

Hall, R.M., Stokes, H.W., 1993. Novel DNA elements which capture genes by site-specific recombination. Genetica 90, 115-132.

Hall, R.M., Vocker, C., 1987. The region of the IncN plasmid R46 coding for resistance to betalactam antibiotics, streptomycin/spectinomycin and sulphonamides is closely related to antibiotic-resistance segments found in IncW plasmids and Tn21-like transposons. Nucleic Acids Res. 15, 7491-7501.

Hall, R.M., Brookes, D.E., Stokes, H.W., 1991. Site-specific insertion of genes into integrons: role of the 59-base element and determination of the crossover point. Mol. Microbiol. 5, 1941-1959.

Jeong, J.Y., Yoon, H.J., Kim, E.S., Lee, Y., Choi, S.H., Kim, N.J., Woo, J.H., Kim, Y.S., 2005. Detection of $q n r$ in clinical isolates of Escherichia coli from Korea. Antimicrob. Agents Chemother. 49, 2522-2524.

Kang, S.-G., Lee, D.-Y., Shin, S.-J., Ahn, J.-M., Yoo, H.-S., 2005. Changes in patterns of antimicrobial susceptibility and class 1 integron carriage among Escherichia coli isolates. J. Vet. Sci. 6, 201-205.

Kim, S., Chun, S.G., Lim, O.Y., Park, M.S., Kang, Y.H., Park, Y.H., Lee, B.K., 2004. Genomic relationship of Salmonella enterica serovar Typhimurium DT104 isolates from Korea and the United States. J. Microbiol. 42, 14-19.

Kwon, H.-J., Park, K.-Y., Yoo, H.-S., Park, J.-Y., Park, Y.-H., Kim, S.-J., 2000. Differentiation of Salmonella enterica serotype gallinarum biotype pullorum from biotype gallinarum by analysis of phase 1 flagellin $\mathrm{C}$ gene $(f l i C)$. . Microbiol. Methods 40, 33-38.

Kwon, H.-J., Kim, T.-E., Cho, S.-H., Seol, J.-G., Kim, B.-J., Hyun, J.-W., Park, K.-Y., Kim, S.-J., Yoo, H.-S., 2002. Distribution and characterization of class 1 integrons in Salmonella enterica serotype Gallinarum biotype Gallinarum. Vet. Microbiol. 89, 303-309.

Kwon, H.-J., Park, K.-Y., Park, J.-Y., Park, Y.-H., Kim, S.-J., Yoo, H.-S., 2004. Distribution and study on single nucleotide polymorphism (SNP) of $s p v R$ gene in Korean isolates of Salmonella. J. Infect. Chemother. 36, 335-340.

Lévesque, C., Brassard, S., Lapointe, J., Roy, P.H., 1994. Diversity and relative strength of tandem promoters for the antibiotic-resistance genes of several integrons. Gene 142, 49-54.
Lévesque, C., Piche, L., Larose, C., Roy, P.H., 1995. PCR mapping of integrons reveals several novel combinations of resistance genes. Antimicrob. Agents Chemother. 39, 185-191.

Martinez-Freijo, P., Fluit, A.C., Schmitz, F.-J., Verhoev, J., Jones, M.E., 1999. Many class 1 integrons comprise stable structures occurring in different species of Enterobacteriaceae isolated from widespread geographic regions in Europe. Antimicrob. Agents Chemother. 43, 686-689.

Martínez-Martínez, L., Pascual, A., Jacoby, G.A., 1998. Quinolone resistance from a transferable plasmid. Lancet 351, 797-799.

Naas, T., Mikami, Y., Imai, T., Poirel, L., Nordmann, P., 2001. Characterization of In53, a class 1 plasmid- and composite transposon-located integron of Escherichia coli which carries an unusual array of gene cassettes. J. Bacteriol. $183,235-249$

National Committee for Clinical Laboratory Standards, 2003. Performance Standards for Antimicrobial Disk Susceptibility Tests. Approved standard M2-A8. NCCLS, Wayne, Pa.

Pai, H., Byeon, J.H., Yu, S., Lee, B.K., Kim, S., 2003. Salmonella enterica serovar Typhi strains isolated in Korea containing a multidrug-resistance class 1 integron. Antimicrob. Agents Chemother. 47, 2006-2008.

Recchia, G.D., Hall, R.M., 1995. Gene cassettes: a new class of mobile element. Microbiology 141, 3015-3027.

Schmidt, F.R.J., Nücken, E.J., Henschke, R.B., 1988. Nucleotide sequence analysis of $2^{\prime \prime}$-aminoglycoside nucleotidyl-transferase ANT (2") from Tn 4000: its relationship with $\operatorname{AAD}\left(3^{\prime \prime}\right)$ and impact on $\operatorname{Tn} 21$ evolution. Mol. Microbiol. 2, 709-717.

Sokurenko, E.V., Chesnokova, V., Dykhuizen, D.E., Ofek, I., Wu, X.R., Krogfelt, K.A, Struve, C., Schembri, M.A., Hasty, D.L., 1998. Pathogenic adaptation of Escherichia coli by natural variation of the FimH adhesin. Proc. Natl. Acad. Sci. 95, 8922-8926.

Sokurenko, E.V., Hasty, D.L., Dykhuizen, D.L., 1999. Pathoadaptive mutations: gene loss and variation in bacterial pathogens. Trends Microbiol. 7, $191-195$.

Tenover, F.C., Filpula, D., Phillips, K.L., Plorde, J.J., 1988. Cloning and sequencing of a gene encoding an aminoglycoside 6'-N-acetyl-transferase from an R factor of Citrobacter diversus. J. Bacteriol. 170, 471-473.

Yang, S.J., Park, K.Y., Seo, K.S., Besser, T.E., Yoo, H.S., Noh, K.M., Kim, S.H., Kim, S., Lee, B.K., Park, Y.H., 2001. Multidrug-resistant Salmonella typhimurium and Salmonella enteritidis identified by multiplex PCR from animals. J. Vet. Sci. 2, 181-188. 